\title{
Long-Term Natural Outcomes of Simple Hemorrhage Associated with Lacquer Crack in High Myopia: A Risk Factor for Myopic CNV?
}

\author{
Bing Liu $\mathbb{D}$, Xiongze Zhang, Lan Mi, Ling Chen, and Feng Wen $(i)$ \\ State Key Laboratory of Ophthalmology, Zhongshan Ophthalmic Center, Sun Yat-sen University, Guangzhou 510060, China \\ Correspondence should be addressed to Feng Wen; wenfeng208@foxmail.com
}

Received 3 September 2017; Revised 6 December 2017; Accepted 26 December 2017; Published 24 January 2018

Academic Editor: Malgorzata Mrugacz

Copyright (C) 2018 Bing Liu et al. This is an open access article distributed under the Creative Commons Attribution License, which permits unrestricted use, distribution, and reproduction in any medium, provided the original work is properly cited.

\begin{abstract}
Purpose. To investigate the relationship between simple hemorrhage (SH) associated with lacquer crack (LC) and myopic choroidal neovascularization (CNV) in high myopia. Methods. A cross-sectional evaluation including best-corrected visual acuity (BCVA), axial length, refractive error, color fundus photography, and spectral domain optical coherence tomography (SD-OCT) was performed in patients diagnosed with high myopia and SH. Fundus fluorescein angiography and indocyanine green angiography were performed if the eye was suspected with CNV. Results. Thirty-three eyes of 27 patients with SH were enrolled in the study. None of the eyes developed $\mathrm{CNV}$ at final examination following the occurrence of hemorrhage. Recurrent hemorrhage was observed in $36.5 \%$ of the eyes. Compared with the initial BCVA, the final BCVA was significantly improved ( $P$ $<0.001)$ and correlated with the integrity of the ellipsoid zone in SD-OCT. There was no significant difference in the final BCVA between group 1 (LC crossed the central fovea) and group 2 (no LC crossed the central fovea) $(P=0.299)$. Conclusions $\mathrm{SH}$ associated with LC is not a risk factor for the development of myopic CNV in patients with high myopia. LCs have little influence on the final BCVA unless the integrity of the ellipsoid zone in the central fovea is disrupted.
\end{abstract}

\section{Introduction}

High myopia is a major cause of visual impairment and legal blindness worldwide, especially in Asian countries [1-3]. Among the various myopic fundus lesions, macular hemorrhage is a common vision-threatening complication in high myopia $[4,5]$. Based on the presence of choroidal neovascularization $(\mathrm{CNV})$, macular hemorrhage can be categorized into two types, hemorrhage secondary to myopic $\mathrm{CNV}$ and simple hemorrhage (SH).

There may be a relationship between $\mathrm{SH}$ and myopic $\mathrm{CNV}$ because the formation of both lesions is related to lacquer cracks (LCs) [6-8]. The occurrence of $\mathrm{SH}$ is generally associated with the formation of new LCs or progression of primary LCs $[5,6,9,10]$. Most myopic CNVs seem to emanate from LCs or at least the areas adjacent to LCs, and the extension of LCs accompanies newly developed myopic CNV [11-14]. Therefore, some retina specialists have hypothesized that $\mathrm{SH}$ may be a risk factor for the development of myopic CNV. Several previous studies have reported the prognosis of $\mathrm{SH}$ in highly myopic eyes but have not investigated the relationship between $\mathrm{SH}$ associated with LC and myopic CNV; in addition, the results of these studies are limited by their short follow-up times $[6,15-17]$.

Thus, this study aims to investigate whether $\mathrm{SH}$ associated with $\mathrm{LC}$ is related to myopic CNV in highly myopic eyes. The long-term visual outcomes of highly myopic eyes with SH in a natural history are also evaluated in this study.

\section{Materials and Methods}

We performed a cross-sectional study. Patients with high myopia and $\mathrm{SH}$ who were referred to the Zhongshan 
Ophthalmic Center in Guangzhou, China, between January 1, 2010, and May 31, 2014, were enrolled. The study protocol was approved by the institutional review board of Zhongshan Ophthalmic Center of Sun Yat-sen University. The study was conducted in accordance with the tenets of the Declaration of Helsinki. Written informed consent was obtained from all patients in this study prior to enrollment.

Pathologic myopia was defined as a refractive error $<-6$ diopters (D) or an axial length $>26.0 \mathrm{~mm}$. The diagnosis of $\mathrm{SH}$ was confirmed by ophthalmoscopic examination and fundus fluorescein angiography (FFA). The exclusion criteria included a history of other ocular disorders, such as dense cataract, glaucoma, diabetic retinopathy or other retinal vascular diseases, and age-related macular degeneration (AMD), and a history of vitreoretinal surgery, which might affect visual acuity. Because this study aimed to determine the natural course of $\mathrm{SH}$ in highly myopic eyes, patients who received any treatments for myopic fundus lesions following SH were also excluded.

Patient demographics, initial best-corrected visual acuity (BCVA), axial length, refractive error, color fundus photography, FFA imaging, and a history of follow-up were recorded from patient medical records.

A cross-sectional evaluation of all patients was performed and included BCVA using a Snellen chart, axial length using IOL Master (Carl Zeiss Meditec, Oberkochen, Germany), refractive error, slit lamp examination, dilated fundus examination by indirect ophthalmoscopy $(+90 \mathrm{D})$, color fundus photography, and spectral domain optical coherence tomography (SD-OCT; Spectralis HRA + OCT, Heidelberg Engineering, Heidelberg, Germany) between June 1, 2017, and June 30, 2017. If the diagnosis of CNV could not be confirmed by SD-OCT (hyperreflective area above the damaged retinal pigment epithelial level with intraretinal fluid/increased foveal thickness and/or serous foveal detachment), FFA and indocyanine green angiography (ICGA) (Spectralis HRA + OCT, Heidelberg Engineering, Heidelberg, Germany) were performed. SD-OCT scanning and analysis of all study eyes were performed by one experienced investigator. Horizontal and vertical SDOCT scans of 6 or $3 \mathrm{~mm}$ were centered on the fovea and the location of SH. The integrity of the ellipsoid zone within the $1 \mathrm{~mm}$ centered fovea was evaluated as continuous or discontinuous. In addition, the eyes were divided into group 1 (LC passed the central fovea) and group 2 (no LC passed the central fovea). Differences in visual acuity between the two groups were investigated.

2.1. Statistical Analysis. Data were processed and analyzed using SPSS 16.0 software (Inc., Chicago, IL, USA). For the analysis, Snellen BCVA data were transformed into equivalent logarithms of the minimum angle of resolution (logMAR) values. To evaluate the differences in visual acuity between group 1 and group 2, we performed independent $t$ tests for continuous variables and chi-square tests for categorical variables. Changes in visual acuity at each follow-up were analyzed using paired $t$-tests. A $P$ value $<0.05$ was considered statistically significant.

\section{Results}

3.1. Patients. We screened 319 files, and 58 patients were eligible. Among these patients, 3 could not be contacted, and 28 refused to participate in this study because of good visual acuity. Thus, 33 eyes of 27 patients with $\mathrm{SH}$ were enrolled in the study. There were no differences in age, gender, or geographic location between those who participated and those who did not participate. The clinical characteristics of the 27 patients are summarized in Table 1 . The study included 13 men and 14 women. The mean duration since the initial visit was $50.4 \pm 16.3$ months, with a range of 36 months to 87 months. For all patients, the clinical diagnosis was made within 1 month from the onset of their visual symptoms, such as blurred vision and a fixed shadow in the front of the eye with or without distorted vision.

\subsection{Patient Clinical Data Collected from Medical Records.} The size of hemorrhages ranged from 0.25 to 1.5 disc diameters (DDs), and all hemorrhages covered the central fovea. The mean hemorrhage duration was $3.0 \pm 1.0$ months, with a range of 1 month to 6 months.

LCs were identified by ophthalmoscopy and FFA in 17 eyes $(51.5 \%)$ at the initial examination. After hemorrhage absorption, LCs were observed in 32 eyes $(97.0 \%)$ at the location of the initial hemorrhage, and the mean BCVA improved to $0.38 \pm 0.26 \operatorname{logMAR}$ (0 to $1.0 \log \mathrm{MAR}$ ) from the initial examination. The difference in BCVA between the two time points was significant $(P<0.001)$.

Recurrent hemorrhages were recorded in 12 eyes (36.4\%) from the medical records. Of these eyes, 3 had 3 recurrent hemorrhages, 1 had 2 recurrent hemorrhages, and 8 had one recurrent hemorrhage. The recurrent hemorrhages almost completely resolved within 4 months.

\subsection{Patient Clinical Data at Cross-Sectional Examination.} The mean refractive error was $-14.7 \pm 4.6 \mathrm{D}$, and the mean axial length was $30.1 \pm 1.4 \mathrm{~mm}$ at the final examination. There were significant differences in both the refractive error and axial length between the initial and final examinations (all $P<0.001$ ).

None of the eyes developed CNV at the final examination following the occurrence of hemorrhage.

At the final examination, LCs were observed in 32 eyes (97.0\%), and 12 (37.5\%) LCs crossed the central fovea. No LC was detected in 1 eye from the initial to the final examination.

Compared with the initial mean BCVA, the final mean BCVA was significantly improved $(0.26 \pm 0.21 \operatorname{logMAR}$; $P<0.001)$. The distributions of the initial and final Snellen VA are shown in Figures 1 and 2. The initial Snellen VA was $20 / 40$ or greater in 6 eyes (18.2\%), 20/200 to 20/40 in 21 eyes (63.6\%), and $20 / 200$ or less in 6 eyes (18.2\%). The final Snellen VA was $20 / 40$ or greater in 26 eyes (78.8\%) and 20/200 to $20 / 40$ in 7 eyes (21.2\%). There was a significant difference in the number of eyes with a Snellen VA of 20/40 or greater between the initial and final visits $(P<0.001)$. Moreover, only two eyes had a worse VA at the final visit 
Table 1: Patient clinical characteristics $(n=27)$.

\begin{tabular}{lc}
\hline Characteristics & \\
\hline Age (years) & $39.0 \pm 8.5$ \\
Male/total $(n, \%)$ & $13 / 27(48.1)$ \\
Bilateral hemorrhage $(n, \%)$ & $6 / 27(22.2)$ \\
Refractive error (diopters) & \\
$\quad$ Initial & $-13.2 \pm 4.3$ \\
$\quad$ Final & $-14.7 \pm 4.6$ \\
Axial length (mm) & \\
$\quad$ Initial & $28.7 \pm 1.3$ \\
$\quad$ Final & $30.1 \pm 1.4$ \\
BCVA (logMAR) & \\
$\quad$ Initial & $0.68 \pm 0.40$ \\
$\quad$ Final & $0.26 \pm 0.21$ \\
Mean duration since initial visit (months) & $50.4 \pm 16.3$ \\
Mean duration of hemorrhage (months) & $3.0 \pm 1.0$ \\
\hline
\end{tabular}

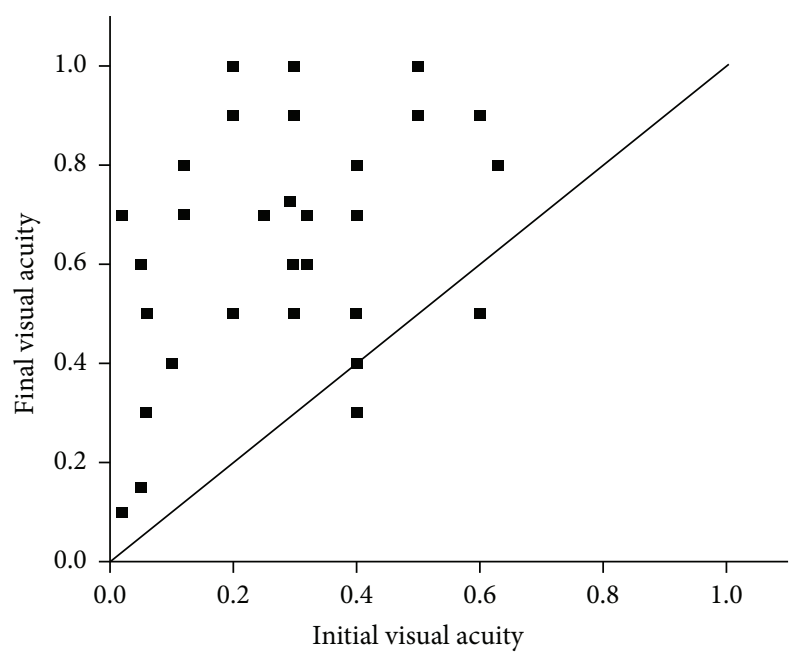

FIgure 1: Initial and final Snellen BCVA of patients in this study. Dots on the line indicate unchanged visual acuity, dots above the line indicate improved visual acuity, and dots below the line indicate worse visual acuity.

because of enlarged retinal pigment epithelium (RPE) and choroid atrophy.

3.4. Relationship between the Final BCVA and Lacquer Cracks. To investigate the relationship between the final BCVA and LCs, we divided 32 eyes with LCs into the following groups: group 1 (LC crossed the central fovea) and group 2 (no LC crossed the central fovea). Although the initial BCVA in group 1 was significantly greater than that in group 2, no significant difference was found in the final BCVA between the two groups (Table 2).

3.5. Relationship between the Final BCVA and OCT Findings. Of the 33 eyes, 27 had a continuous ellipsoid zone in the central fovea in OCT. Compared with eyes with a discontinuous ellipsoid zone, eyes with a continuous ellipsoid zone had a

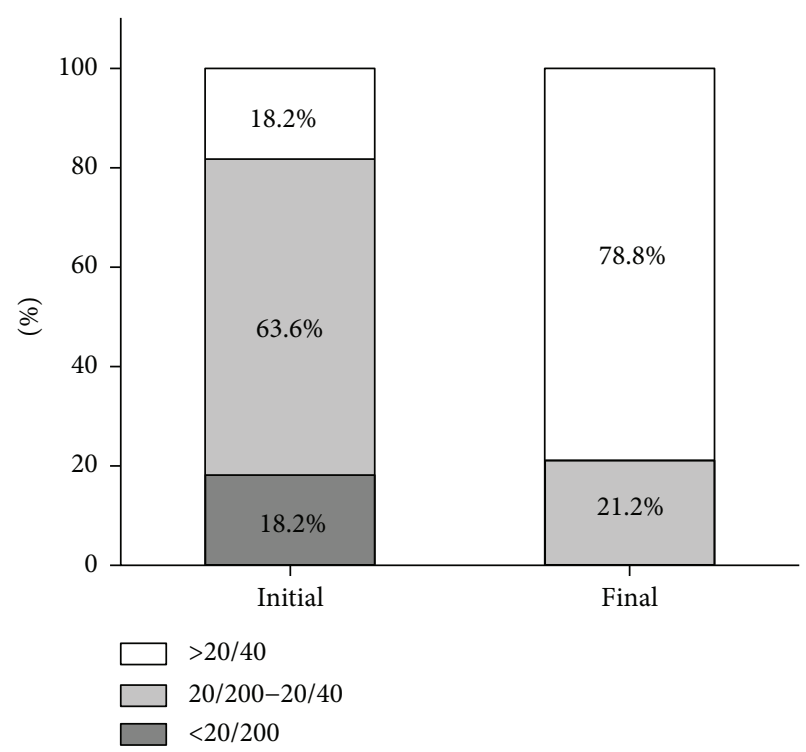

Figure 2: Distribution of the initial and final Snellen BCVA in this study.

TABLE 2: Comparison of clinical characteristics between group 1 and group 2 .

\begin{tabular}{lccc}
\hline & Group 1 & Group 2 & $P$ \\
\hline Eyes & 12 & 20 & - \\
Age (year) & $38.7 \pm 8.1$ & $38.1 \pm 8.1$ & 0.968 \\
Initial refractive error (diopters) & $-13.0 \pm 4.6$ & $-13.4 \pm 4.3$ & 0.830 \\
Initial axial length (mm) & $28.3 \pm 1.1$ & $28.8 \pm 1.5$ & 0.339 \\
Initial BCVA (logMAR) & $0.76 \pm 0.45$ & $0.58 \pm 0.26$ & 0.017 \\
Final BCVA (logMAR) & $0.30 \pm 0.24$ & $0.18 \pm 0.14$ & 0.299 \\
Eyes with recurrent & $5(41.7 \%)$ & $7(35.0 \%)$ & 0.800 \\
hemorrhage $(n, \%)$ & & & \\
\hline
\end{tabular}

significantly improved final BCVA $(0.48 \pm 0.35 \log$ MAR versus $0.21 \pm 0.13 \log$ MAR; $P<0.001$, Figures 3 and 4 ).

\section{Discussion}

$\mathrm{SH}$ is a term used for macular hemorrhage without $\mathrm{CNV}$ that occurs in highly myopic eyes and is most likely caused by the mechanical rupture of Bruch's membrane and choriocapillaris complex $[15,16]$. Although some researchers have suspected that SH may be a precursor of myopic CNV [15], the evidence is insufficient. A better understanding of the predictors of myopic CNV development may lead to more personalized treatment and help improve clinical outcomes and reduce recurrence $[8,18]$. To investigate the relationship between $\mathrm{SH}$ associated with $\mathrm{LC}$ and myopic CNV, we performed a cross-sectional study.

In this study, no eyes with $\mathrm{SH}$ associated with LC developed CNV following hemorrhage occurrence. This result is consistent with the results of several previous studies [15, 16]. Goto et al. examined 20 eyes of 17 consecutive patients with $\mathrm{SH}$ and found that most of the patients had a good recovery, and no eye in the $\mathrm{SH}$ group developed $\mathrm{CNV}$ during 


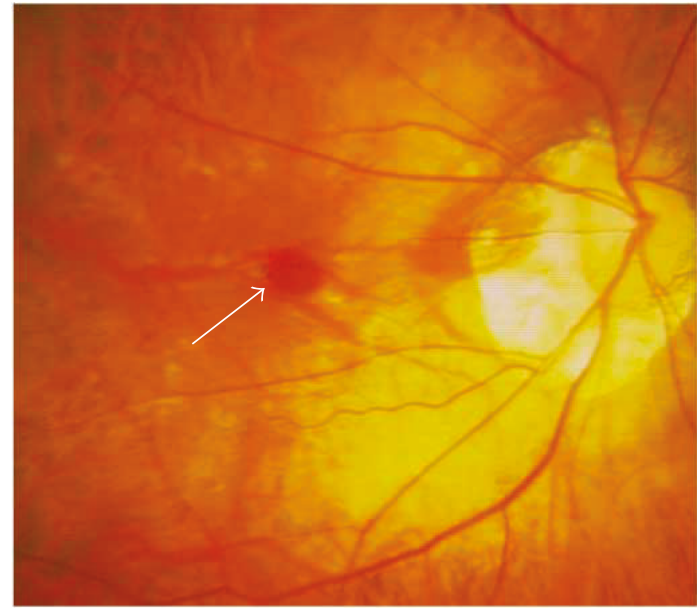

(a)

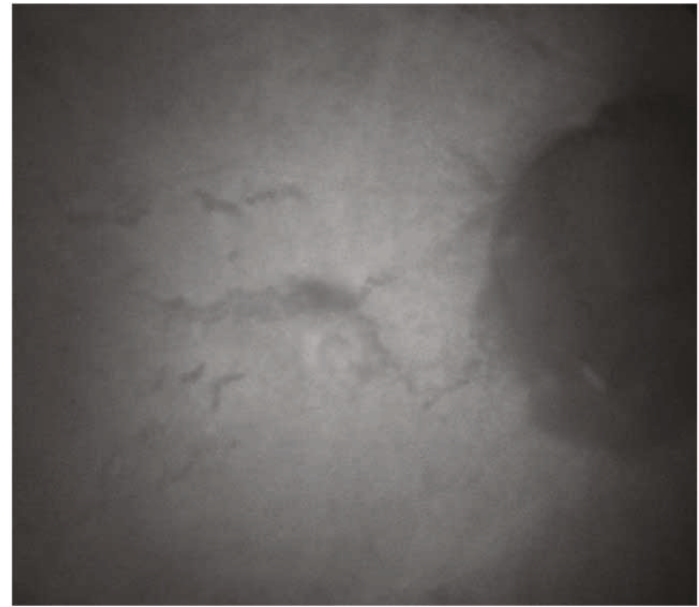

(c)

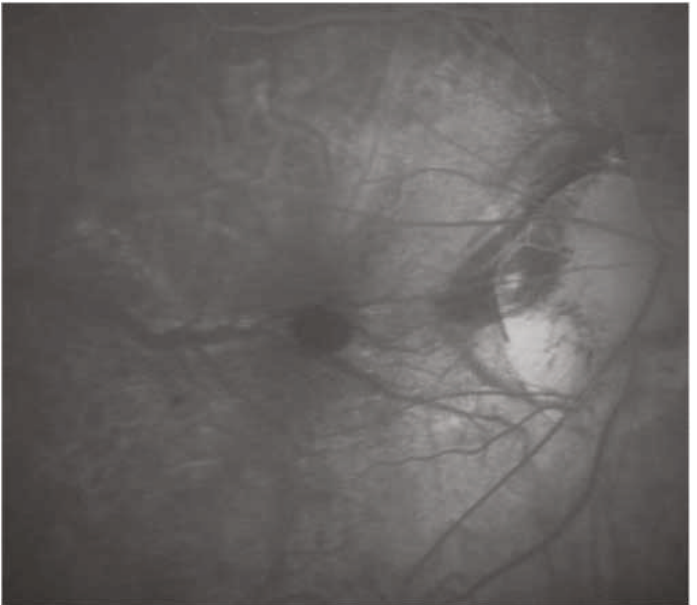

(b)

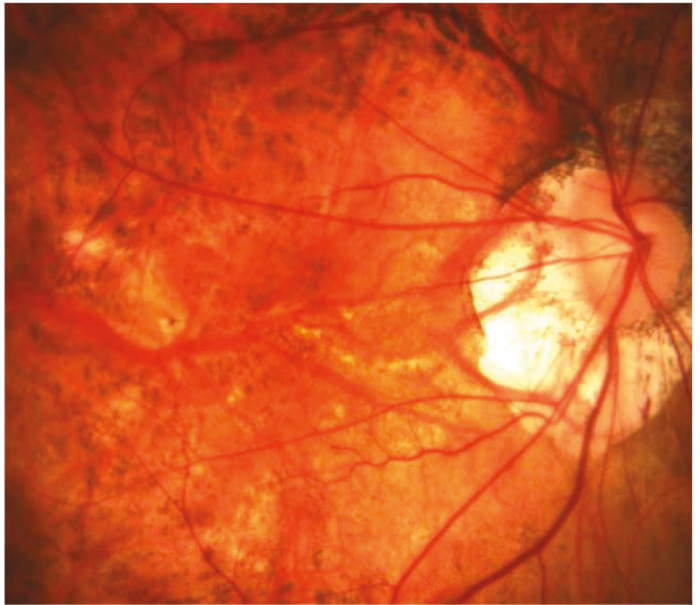

(d)

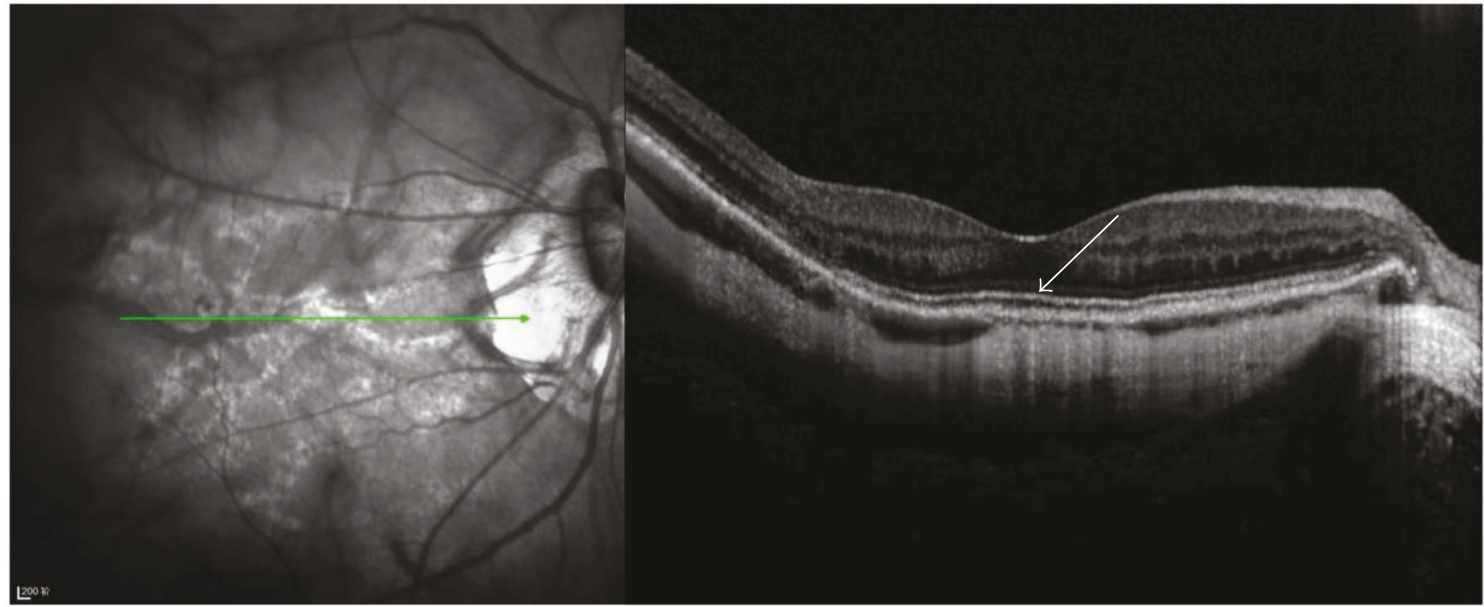

(e)

FIGURE 3: Multimodal imaging of an eye with a continuous ellipsoid zone. A 52-year-old woman was initially seen on September 2, 2011, with decreased visual acuity and a fixed shadow in her right eye. At the initial examination, the BCVA was 20/100, the refractive error was -8.75 D, and the axial length was $29.7 \mathrm{~mm}$ in her right eye. There was subretinal hemorrhage (white arrow) in the macular area of the right eye at the initial examination (a). The late phases of FFA and ICGA revealed a simple hemorrhage (b and c). During the follow-up period, recurrent hemorrhage and CNV were not detected. At the final examination (June 2, 2017), LCs were observed in the macular area by color fundus photography (d). An LC passing through the central fovea was easily observed with near-infrared reflectance imaging (e). Because the ellipsoid zone (white arrow) at the central fovea was continuous (e), the final BCVA of the eye was still good (20/25). 


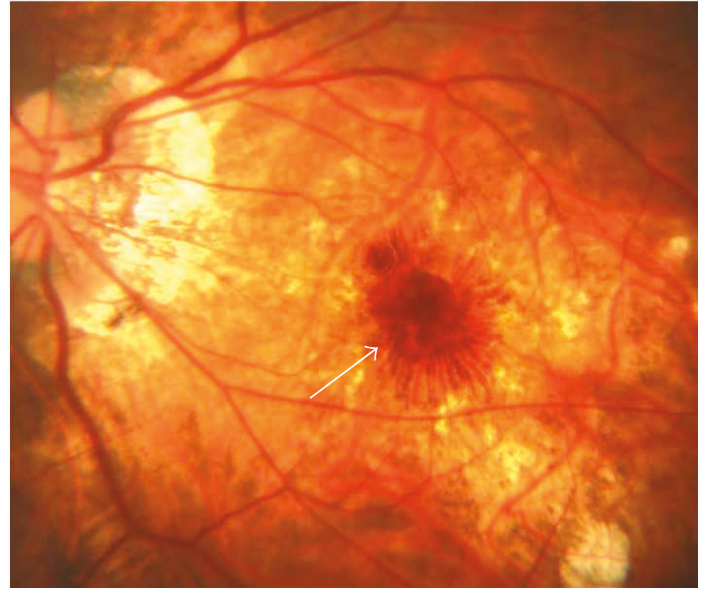

(a)

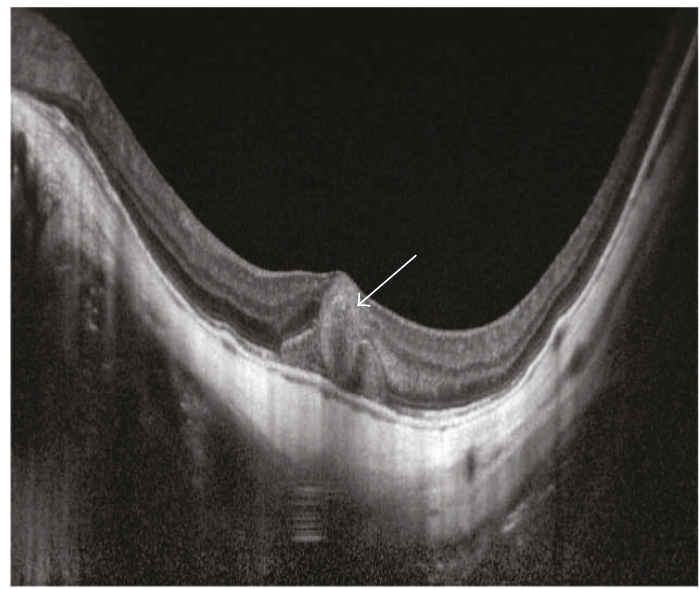

(c)

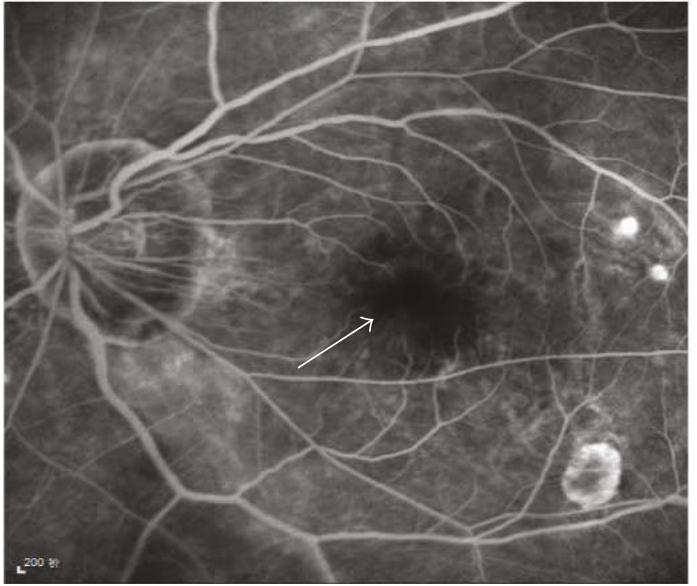

(b)

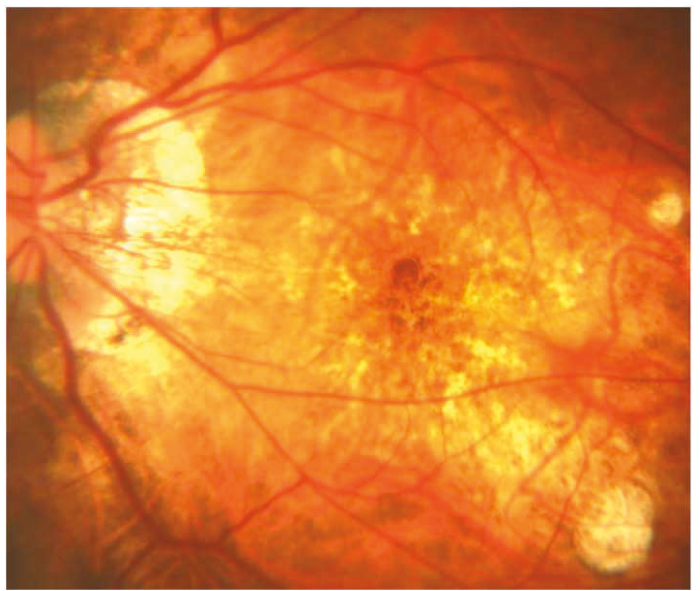

(d)

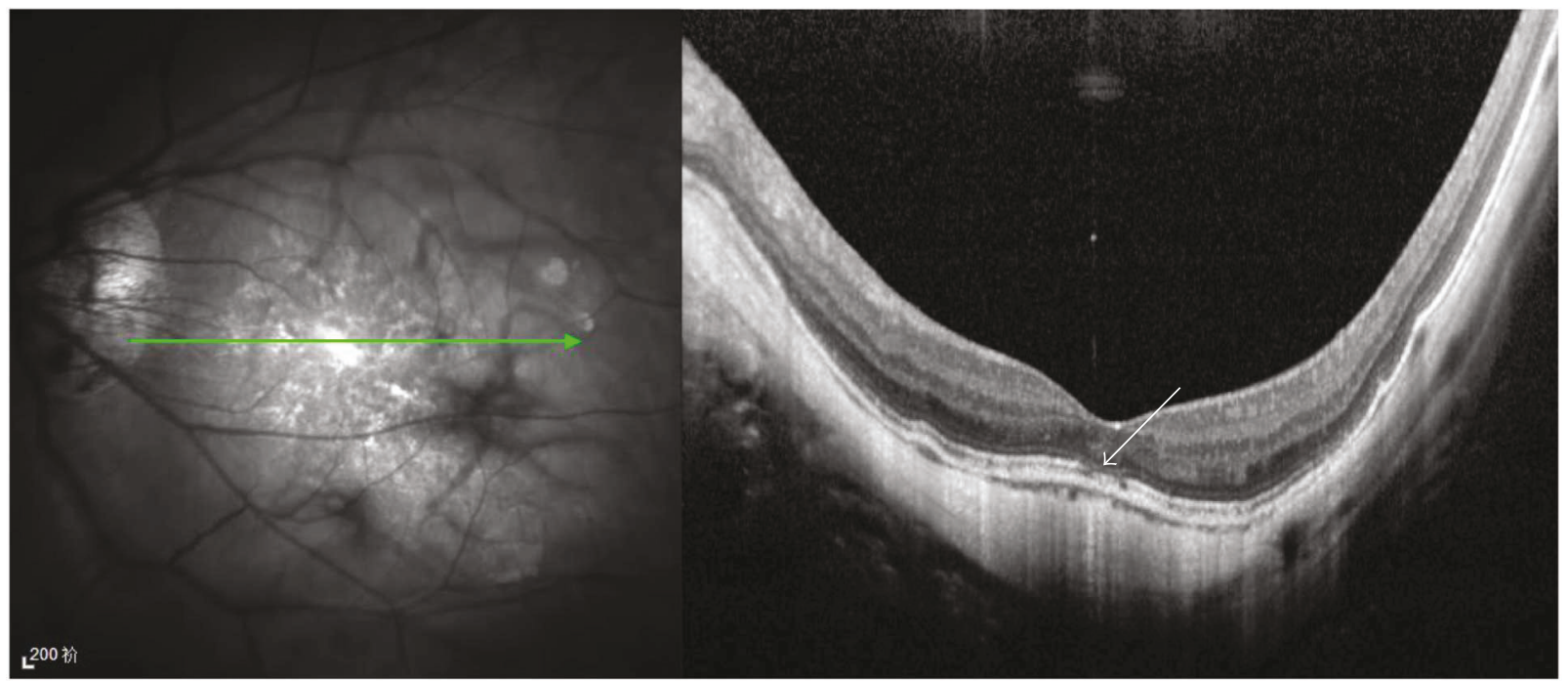

(e)

FIGURE 4: Multimodal imaging of an eye with a discontinuous ellipsoid zone. A 40-year-old woman was initially seen on December 3, 2013, with decreased visual acuity in her left eye. At the initial examination, the BCVA was $5 / 100$, the refractive error was $-16.5 \mathrm{D}$, and the axial length was $27.37 \mathrm{~mm}$ in her left eye. An obvious subretinal hemorrhage (white arrow) was observed in the macular area at the initial examination (a). The late phase of FFA indicated that it was a simple hemorrhage (white arrow) (b). SD-OCT showed that the hemorrhage (white arrow) was thick and reached beyond the ellipsoid zone (c). After 3 years of follow-up, no recurrent hemorrhage or $\mathrm{CNV}$ occurred. Because the ellipsoid zone (white arrow) at the central fovea was not continuous (e), the final BCVA of the eye was poor $(20 / 100)$. 
the 1-year follow-up [15]. Moriyama et al. examined 31 eyes of 28 patients with high myopia and $\mathrm{SH}$; they also found that no eye developed CNV during a mean follow-up of 17.7 months [16]. These results may be explained by differences in the pathogenesis between $\mathrm{SH}$ associated with LC and myopic CNV. LCs are caused by the mechanical rupture of Bruch's membrane. If both the choriocapillaris and Bruch's membrane rupture during formation or progression of LCs, then $\mathrm{SH}$ could be observed. The pathogenesis of myopic $\mathrm{CNV}$ remains controversial, and several theories, such as the mechanical theory, heredodegenerative theory, and hemodynamic changes in choroidal circulation [19-23], have been proposed. In our opinion, the development of myopic CNV may result from the interaction of multiple factors. LCs are necessary but not sufficient for the development of myopic $\mathrm{CNV}$. The occurrence of myopic CNV may require other conditions mentioned above. Therefore, $\mathrm{SH}$ associated with $\mathrm{LC}$ is not a risk factor for the development of myopic CNV.

The visual prognosis of the myopic eyes with $\mathrm{SH}$ may be fair during a short follow-up period unless the hemorrhage recurs, atrophic scars develop, or retinochoroidal degeneration progresses [17]. In this study, similar results were observed in the long-term natural history. Most patients had good visual outcomes after at least 3 years of observation except for two eyes in which severe diffuse retinochoroidal atrophy was detected in the posterior fundus. In addition, recurrent hemorrhages were detected in 12 eyes (36.4\%), indicating a high prevalence of recurrent hemorrhage in highly myopic eyes.

In this study, we also evaluated the relationship between final visual acuity and fundus changes. There was a significant correlation between the visual outcome and OCT findings. At the last visit, eyes with a continuous ellipsoid zone in the central fovea usually had better visual outcomes. This result is compatible with the findings of previous reports $[15,16,24,25]$. For eyes with a discontinuous ellipsoid zone, the SH may be thick and reach beyond the ellipsoid zone; thus, severe damage to the retinal structure may limit visual recovery after hemorrhage absorption [16]. In addition, LCs had little effect on the final visual acuity when they crossed the central fovea. This finding is interesting and has not been previously reported. However, our OCT findings can explain this result. LCs are formed by ruptures in Bruch's membrane in which small hemorrhages may develop. After the hemorrhages were absorbed, there were usually no special findings in the layers of the RPE, Bruch's membrane, and choriocapillaris complex at the location of LCs in OCT. If the ellipsoid zone above the LCs was continuous, the eye with $\mathrm{SH}$ had a good visual outcome; otherwise, the visual acuity was poor. Thus, ellipsoid zone integrity but not LCs reflected the final outcomes of visual acuity. In addition, although 12 eyes had recurrent hemorrhages, their final visual acuity remained good. We found that these eyes had a continuous or relatively continuous ellipsoid zone in the central fovea in OCT. Thus, these findings also revealed the importance of the integrity of the ellipsoid zone in the central fovea for the final visual acuity.

Our study has several limitations that need to be considered. First, all patients were from a single institution; thus, a referral bias may exist. Second, a small number of patients participated in this study. However, to the best of our knowledge, this study is the first to evaluate the relationship between $\mathrm{SH}$ associated with $\mathrm{LC}$ and myopic CNV in high myopia in a long-term natural history.

In conclusion, in this study, we demonstrated that $\mathrm{SH}$ associated with LC is not a risk factor for the development of myopic $\mathrm{CNV}$ and that $\mathrm{SH}$ may recur in approximately $1 / 3$ of eyes with high myopia. The long-term visual outcome of $\mathrm{SH}$ in high myopia was generally good, and it correlated with the integrity of the ellipsoid zone in OCT. In addition, LCs that passed through the central fovea had little influence on the final visual acuity unless the integrity of the ellipsoid zone in the central fovea was disrupted.

\section{Conflicts of Interest}

The authors declare no conflicts of interest.

\section{Acknowledgments}

This research was supported by the National Natural Science Foundation of China (Grant no. 81570831) and the Fundamental Research Funds of State Key Laboratory of Ophthalmology.

\section{References}

[1] L. Xu, Y. Wang, Y. Li et al., "Causes of blindness and visual impairment in urban and rural areas in Beijing: the Beijing Eye Study," Ophthalmology, vol. 113, no. 7, pp. 1134.e11134.e11, 2006.

[2] A. Iwase, M. Araie, A. Tomidokoro et al., "Prevalence and causes of low vision and blindness in a Japanese adult population: the Tajimi study," Ophthalmology, vol. 113, no. 8, pp. 1354-1362.e1, 2006.

[3] X. He, R. Zhao, P. Sankaridurg et al., "Design and methodology of the Shanghai child and adolescent large-scale eye study (SCALE)," Clinical \& Experimental Ophthalmology, 2017.

[4] H. Chen, F. Wen, H. Li et al., "The types and severity of high myopic maculopathy in Chinese patients," Ophthalmic \& Physiological Optics, vol. 32, no. 1, pp. 60-67, 2012.

[5] K. Hayashi, K. Ohno-Matsui, N. Shimada et al., "Long-term pattern of progression of myopic maculopathy: a natural history study," Ophthalmology, vol. 117, no. 8, pp. 15951611.e4, 2010.

[6] K. Ohno-Matsui, M. Ito, and T. Tokoro, "Subretinal bleeding without choroidal neovascularization in pathologic myopia: a sign of new lacquer crack formation," Retina, vol. 16, no. 3, pp. 196-202, 1996.

[7] R. M. Klein and S. Green, "The development of lacquer cracks in pathologic myopia," American Journal of Ophthalmology, vol. 106, no. 3, pp. 282-285, 1988.

[8] K. Ohno-Matsui, T. Y. Y. Lai, C.-C. Lai, and C. M. G. Cheung, "Updates of pathologic myopia," Progress in Retinal and Eye Research, vol. 52, pp. 156-187, 2016.

[9] L. W. Yip and K.-G. Au Eong, "Recurrent subretinal haemorrhages and progressive lacquer cracks in a high myope," Acta Ophthalmologica Scandinavica, vol. 81, no. 6, pp. 646647, 2003. 
[10] K. Ohno-Matsui and T. Tokoro, "The progression of lacquer cracks in pathologic myopia," Retina, vol. 16, no. 1, pp. 2937, 1996.

[11] R. Axer-Siegel, D. Cotlear, E. Priel, I. Rosenblatt, M. Snir, and D. Weinberger, "Indocyanine green angiography in high myopia," Ophthalmic Surgery, Lasers and Imaging, vol. 35, pp. 139-145, 2004.

[12] Y. Ikuno, K. Sayanagi, K. Soga et al., "Lacquer crack formation and choroidal neovascularization in pathologic myopia," Retina, vol. 28, no. 8, pp. 1124-1131, 2008.

[13] Y. M. Kim, J. U. Yoon, and H. J. Koh, "The analysis of lacquer crack in the assessment of myopic choroidal neovascularization," Eye, vol. 25, no. 7, pp. 937-946, 2011.

[14] H. M. Kang and H. J. Koh, "Ocular risk factors for recurrence of myopic choroidal neovascularization: long-term follow-up study," Retina, vol. 33, no. 8, pp. 1613-1622, 2013.

[15] S. Goto, K. Sayanagi, Y. Ikuno, Y. Jo, F. Gomi, and K. Nishida, "Comparison of visual prognoses between natural course of simple hemorrhage and choroidal neovascularization treated with intravitreal bevacizumab in highly myopic eyes: a 1-year follow-up," Retina, vol. 35, no. 3, pp. 429-434, 2015.

[16] M. Moriyama, K. Ohno-Matsui, N. Shimada et al., "Correlation between visual prognosis and fundus autofluorescence and optical coherence tomographic findings in highly myopic eyes with submacular hemorrhage and without choroidal neovascularization," Retina, vol. 31, no. 1, pp. 74-80, 2011.

[17] S. Hayasaka, M. Uchida, and T. Setogawa, "Subretinal hemorrhages with or without choroidal neovascularization in the maculas of patients with pathologic myopia," Graefe's Archive for Clinical and Experimental Ophthalmology, vol. 228, no. 4, pp. 277-280, 1990.

[18] N. Leveziel, D. Gaucher, S. Baillif et al., "Understanding the determinants of myopic choroidal neovascularization and response to treatment," European Journal of Ophthalmology, vol. 26, no. 6, pp. 511-516, 2016.

[19] K. Neelam, C. M. G. Cheung, K. Ohno-Matsui, T. Y. Y. Lai, and T. Y. Wong, "Choroidal neovascularization in pathological myopia," Progress in Retinal and Eye Research, vol. 31, no. 5, pp. 495-525, 2012.

[20] T. Wakabayashi and Y. Ikuno, "Choroidal filling delay in choroidal neovascularisation due to pathological myopia," The British Journal of Ophthalmology, vol. 94, no. 5, pp. 611-615, 2010.

[21] N. Leveziel, Y. Yu, R. Reynolds et al., "Genetic factors for choroidal neovascularization associated with high myopia," Investigative Ophthalmology \& Visual Science, vol. 53, no. 8, pp. 5004-5009, 2012.

[22] Y. Akagi-Kurashige, K. Kumagai, K. Yamashiro et al., "Vascular endothelial growth factor gene polymorphisms and choroidal neovascularization in highly myopic eyes," Investigative Ophthalmology \& Visual Science, vol. 53, no. 4, pp. 23492353, 2012.

[23] M. Miyake, K. Yamashiro, H. Nakanishi et al., "Evaluation of pigment epithelium-derived factor and complement factor I polymorphisms as a cause of choroidal neovascularization in highly myopic eyes," Investigative Ophthalmology \& Visual Science, vol. 54, no. 6, pp. 4208-4212, 2013.
[24] T. Asai, Y. Ikuno, and K. Nishida, "Macular microstructures and prognostic factors in myopic subretinal hemorrhages," Investigative Ophthalmology \& Visual Science, vol. 55, no. 1, pp. 226-232, 2014.

[25] P. Milani, M. Pellegrini, A. Massacesi et al., "Is ellipsoid zone integrity essential for visual recovery in myopic neovascularization after anti-VEGF therapy?," Graefe's Archive for Clinical and Experimental Ophthalmology, vol. 255, no. 9, pp. 17131720, 2017. 


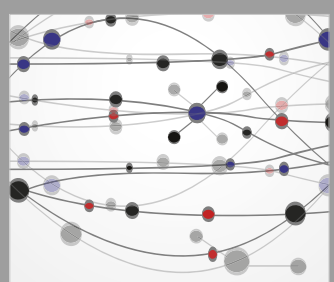

The Scientific World Journal
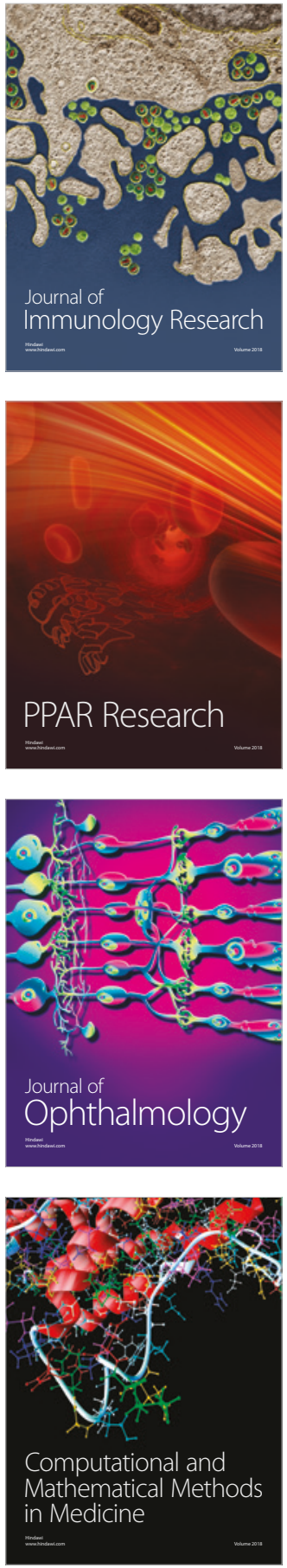

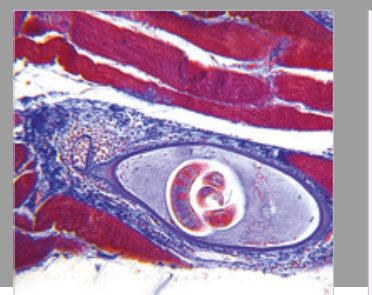

Gastroenterology Research and Practice

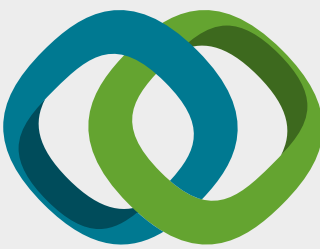

\section{Hindawi}

Submit your manuscripts at

www.hindawi.com
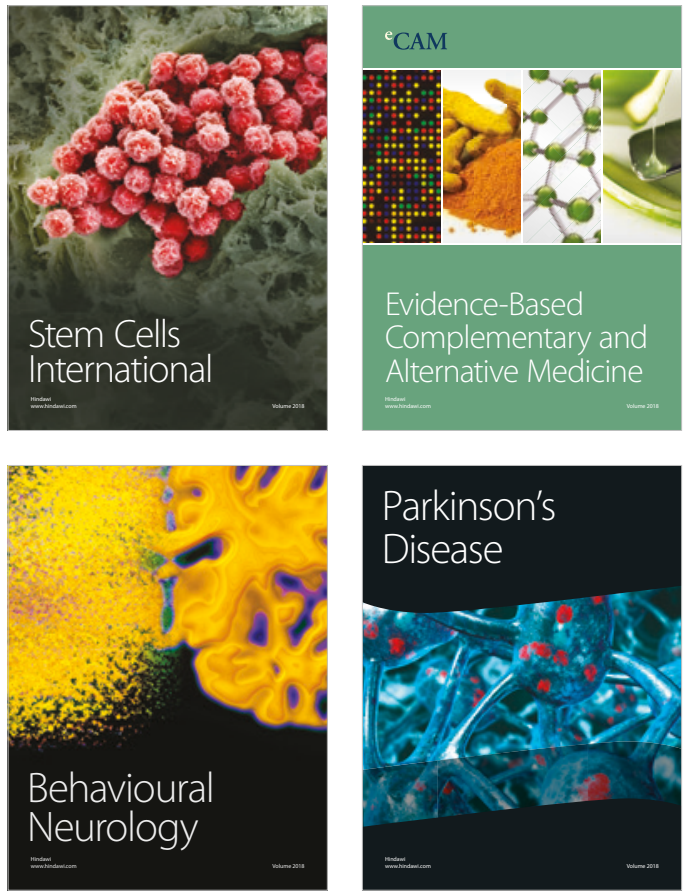

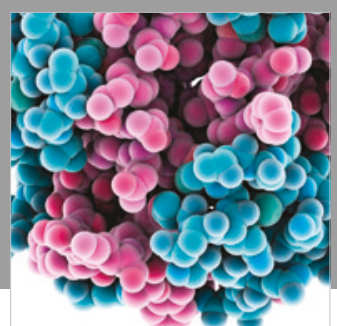

ournal of

Diabetes Research

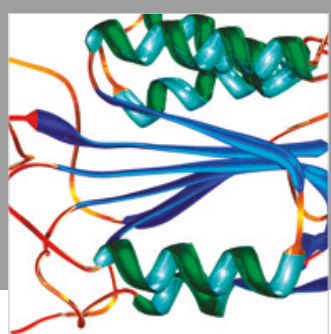

Disease Markers
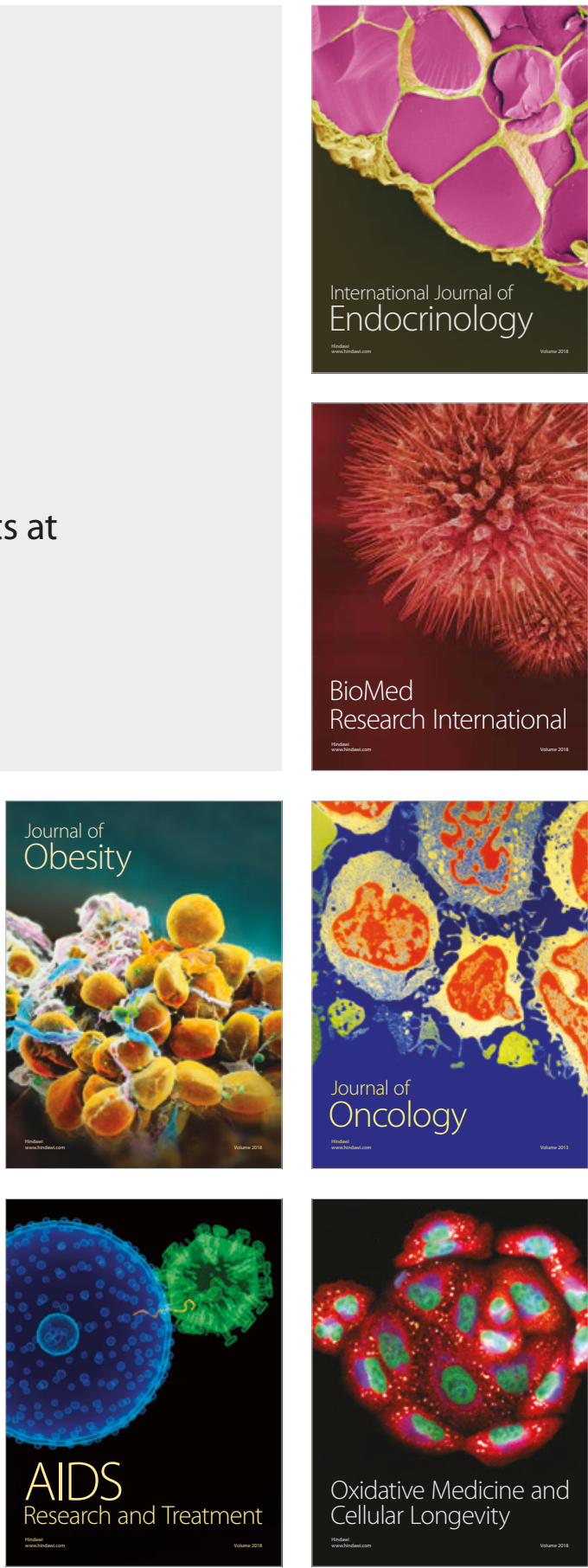